\title{
Peter Landau
}

\section{Das Weihehindernis der Illegitimität in der Geschichte des kanonischen Rechts}

\section{Einleitung}

Das kanonische Recht hat als wesentlichen Gegenstand seit frühesten Zeiten den Bereich der Ordination, deren rituelle und wohl auch in einer Art von Rechtsbewußtsein gefestigte Ursprünge schon im ersten Jahrhundert liegen. Für die Ordination sind bereits frühzeitig Qualifikationserfordernisse bei den Kandidaten gefordert worden, die im Neuen Testament zuerst in den sogenannten Pastoralbriefen aufgezählt werden. ${ }^{1}$ Hier liegt der Anfang einer Entwicklung sogenannter Irregularitätsbestimmungen, die dann im Recht der mittelalterlichen Kirche eine zentrale Rolle gespielt haben. Man konnte zum geistlichen Dienst aufgrund von Charakterfehlern ungeeignet sein, aber auch aufgrund von objektiv vorhandenen Defekten, da der Ausschluß von der Ordination von Anfang an nicht als Sanktion verstanden wurde. Als solche Defekte, die nichts mit Charakterfehlern zu tun haben; nennt bereits der erste Timotheusbrief, daß man vom Bischofs- und Diakonsamt ausgeschlossen sei, wenn man zweimal im Leben verheiratet war, und daß außerdem Neubekehrte (Neophyten) nicht Bischöfe werden sollten. ${ }^{2}$ Bereits aus der Formulierung in den Pastoralbriefen läßt sich erkennen, daß der Ausschluß bestimmter Personen vom geistlichen Dienst mit Gesichtspunkten des Ansehens der Gemeinde in der nichtchristlichen Umwelt zusammenhängt - das Christentum will durch seine Botschaft Anstoß erregen, aber nicht durch die Person seiner Repräsentanten.

Dieser Grundgedanke steht auch hinter dem Prinzip des kanonischen Rechts, illegitim Geborene nicht zum Klerus zuzulassen. Die entsprechenden Rechtssätze sind allerdings lange Zeit in der Kirche nicht vorhanden gewesen, sondern erst nach etwa einem Jahrtausend im 10. und 11. Jahrhundert im Ansatz ausgebildet worden - und sie sind auch heute, anders als etwa das in derselben Zeit in der Kirche durchgesetzte Zölibatsgebot, wiederum aus dem kanonischen Recht verschwunden. Noch der Codex von 1917 nennt als erste der irregularitates ex defectu: Illegitimi, sive illegitimitas sit publica sive occulta, nisi

1 1. Tim. 3.1-13 und Tit. 1.5-9. Zu den Pastoralbriefen vgl. Jean Dauvillier, Les temps apostoliques (Hist. du Droit et des Inst. de l'Eglise 2, Paris 1970) 65-67 mit Uberblick über die sehr kontroverse Literatur. Vgl. auch Heinrich Schlier, Die Ordnung der Kirche nach den Pastoralbriefen, in: ders., Die Zeit der Kirche (Freiburg 3. Aufl. 1962) 129-147; Hans-Werner Bartsch, Die Anfänge urchristlicher Rechtsbildungen. Studien zu den Pastoralbriefen (Hamburg 1965).

1. Tim. 3.2 und 3.6. 
fuerint legitimati vel vota sollemnia professi ${ }^{3}$; der $\mathrm{CIC}$ von 1983 hat diese wie auch sonstige Irregularitäten ex defectu (Körpermängel, Abstammung von Ketzern, Sklavenstatus) nicht mehr übernommen. ${ }^{4}$ Es handelt sich hier also nach der Auffassung der Kommission, die den jetzt geltenden Codex redigierte, nicht um ein aus der Bibel oder nach der Tradition der Kirche zwingend begründetes Verbot. Die historische Ausbildung des Verbots soll hier in einer Skizze erfaßt werden.

Zunächst ist festzuhalten, daß in keiner einzigen Rechtsvorschrift des spätantiken Kirchenrechts ein Ausschluß von der Weihe wegen eines auf der Geburt von Personen haftenden Makels ${ }^{5}$ festgesetzt wird. Ganz im Gegenteil - es gibt eine Reihe von Aussprüchen der Kirchenväter, wonach die Geburt aus einem ehebrecherischen oder sonst unreinen Verhältnis niemandem als Schuld angerechnet werden dürfe. In diesem Zusammenhang gibt es vor allem Textstellen bei Johannes Chrysostomus, Hieronymus und Augustinus; so erklärt Hieronymus in einem auch von Gratian zitierten Brief: Nasci de adulterio non est eius culpa, qui nascitur, sed illius, qui generat (56.5). ${ }^{6}$ Schon die rechtliche Anerkennung des Konkubinats mußte zur Folge haben, daß Konkubinenkinder auch in der Kirche nicht benachteiligt wurden.

\section{Karolingische Anfänge}

Die Anfänge der Irregularität wegen des defectus natalium liegen in der Karolingerzeit; sie sind eine Folge der karolingischen Bemühungen um Kirchenreform, zu denen auch das Streben nach einem hohen Standard des Klerus gehörte. Eine erste in diese Richtung weisende Bestimmung erläßt $845 / 46$ das Konzil von Meaux-Paris, wo es in c. 64 heißt, daß die nach einer Entführung zwischen dem Entführer und der Entführten vor einer nachmals zwischen ihnen formalisierten Eheschließung erzeugten Söhne prinzipiell keine kirchlichen Weihen erhalten sollen, es sei denn, daß Nutzen oder Notwendigkeit der Kirche eine Ausnahme nahelege. ${ }^{7}$ Wilfried Hartmann hat diesen Kanon in seiner Edition zutreffend kommentiert: „Überhaupt war es im Kirchenrecht völlig neu, jemanden wegen eines Mangels seiner Geburt von der Priesterweihe auszuschließen. ${ }^{\text {"8 }}$ Der Kanon wurde in

3 Can. 984, Zif. $1 \mathrm{CIC} / 1917$.

4 Zur Aufgabe der Unterscheidung von Weihehindernissen ex defectu und ex delicto im Codex Iuris Canonici von 1983 vgl. Hubert Müller, in: Handbuch des katholischen Kirchenrechts. Hrsg. v. Joseph Listl, Hubert Müller, Heribert Schmitz (Regensburg 1983) 727 Anm. 92 (\$79); ferner Communicationes pontifica commissio codici iuris canonici recognoscendo 10 (Rom 1978) 196.

3 So Paul Hinschius, System des katholischen Kirchenrechts, Bd. 1 (Berlin 1869, ND Graz 1959) 11.

6 Die entsprechenden Texte erscheinen bei Gratian in 56.3-9. Diese Kirchenväterstellen kamen erst gegen Ende des 11. Jahrhunderts in die kanonistische Tradition, meist über die Sammlungen des Ivo von Chartres. Das bedeutet, daß man offenbar solche Kirchenväterstellen erst zusammenstellte, als bereits in der konziliaren Gesetzgebung der Ausschluß der Illegitimen akut geworden war.

7 Conc. Meldense ao. 845/846 c. 64. Hrsg. v. Wilfried Hartmann, MGH Conc. 3 (künftig: MGH Conc.

3) 115: Filii vero ex buiusmodi vituperabili coniunctione ante coniugium etiam minus laudabile procreati ad ecclesiasticam dignitatem nullo modo provehantur; nec de tali coniugio generati ecclesiasticis ordinibus applicentur, nisi forte cos aut maxima ecclesie utilitas vel necessitas postulet vel evidens meritorum praerogativa commendet.

8 MGH Conc. 3 (wie Anm. 7), 115 Anm. 193. 
der späteren kanonistischen Tradition vor allem von Regino von Prüm rezipiert, aber nicht von Burchard von Worms oder Ivo von Chartres; ${ }^{9}$ Gratian hat ein Exzerpt, das er über Alger von Lüttich kannte, für ein Dictum seines Dekrets verwandt (1.7.16). ${ }^{10}$ Wichtig ist die dem Kanon beigegebene Begründung - die Söhne aus einer solchen Entführungsverbindung gelten als ex huiusmodi vituperabili coniunctione minus laudabile procreati - entgegen der Auffassung der Kirchenväter führt der Defekt der Verbindung der Eltern auch zu einem Defekt des Sprößlings. ${ }^{11}$ Regino von Prüm greift zu Beginn des 10. Jahrhunderts in seinem Sendhandbuch den Kanon von Meaux auf und verbindet ihn mit einem falsch gedeuteten Kanon des Konzils von Orléans 538 sowie einer über das Breviar rezipierten Stelle der Institutionen des Gaius. ${ }^{12}$ Die drei Texte werden von Regino unter die gemeinsame Rubrik gestellt: De filiis concubinarum, ne ad clerum admittantur. ${ }^{13}$

Interessant ist in diesem Zusammenhang auch, daß Regino die Gaiusstelle mit einem eigenen ergänzenden Kommentar verknüpft. ${ }^{14}$ Gaius hatte festgestellt, daß Söhne aus einer verbotenen und inzestuösen Ehe Ehebruchskindern gleichzustellen seien, folglich als unehelich gelten müßten. Regino fügt nun als offenbar eigenes Produkt die Schlußfolgerung hinzu, daß solche in der weltlichen Rechtsordnung nach seiner Ansicht mit dem Makel der Infamie behafteten Kinder auch keine kirchlichen Würden erhalten dürften praesertim cum nihil maculosum Deo rite possit offerri vel eius servitio applicari. ${ }^{15}$ Diese Begründung weist deutlich darauf hin, daß die enge Beziehung des Priesters zum Meßopfer ein Hauptgrund für die Entwicklung des Weiheverbots bei Nichtehelichen war. Man kann daher sagen, daß dieses Verbot zuerst klar bei Regino von Prüm formuliert ist, was in der kirchenrechtlichen Literatur bisher nicht erkannt wurde, die seit Thomassin im 17. Jahrhundert generell die Entstehung des Verbots auf die Reformkonzilien des 11. Jahrhunderts zurückführt. ${ }^{16}$ Regino war hier wie wohl auch in anderen Bereichen ein Neuerer, was in der Forschung bisher nicht ausreichend erkannt wurde, da man keinen genauen Überblick über Interpolationen und Zusätze in den von Regino rezipierten

9 Vgl. MGH Conc. 3 (wie Anm. 7), 74: Regino, Libri duo de synodalibus causis 1. 428. Hrsg. v. Friedrich Wilhelm Hermann Wasserschleben (Leipzig 1840, ND Graz 1964) 193f. Im folgenden zitiert: Regino, Libri bzw. Wasserschleben, Regino.

10 D.p. 1.7.16: Utilitatis intuitu: sicut in Meldensi Concilio, perhiberur de filiis uirginum per uim raptarum et sic ad coniugium raptorum applicatarum. Quelle Gratians ist Alger, De misericordia et iustitia I, 15. Robert Kretzschmar (Hrsg.), Alger von Lüttichs Traktat „De misericordia et iustitia“, Sigmaringen 1985 (Quellen und Forsch. zum Recht im Mittelalter 2) 201.

11 MGH Conc. 3 (wie Anm. 7), 115.

12 Conc. Aurelianense ao. 538 c. 10. Hrsg. v. Carolus de Clercq, Conciliae Galliae A. 511-695, Turnholt 1963 (CC SL 148A) 118 = Regino, Libri (wie Anm. 9), 1.427, 193; Brev. Gaii Inst. IV, 8. Ed. by Gustav Haenel, Lex Romana Visigothorum, 318 = Regino, Libri (wie Anm. 9), 1.429, 194.

13 Rubrik zu Regino, Libri (wie Anm. 9), 1.427, 193.

14 Regino, Libri (wie Anm. 9), 1.429 ab Eos itaque - servitio applicari. Vgl. Wasserschleben, Regino (wie Anm. 9), 194 Anm. 690: Sed, quae iam sequuntur, nec in Gaio, nec alias sunt reperta.

${ }_{15}$ Regino, Libri (wie Anm. 9), 1.429, 194 am Ende.

16 Louis de Thomassin, Ancienne et nouvelle Discipline de l'Eglise, Bd. 3 (Paris 1681), P. IV, L. II, ch. XXIX (106-111), die erste rechtshistorische Darstellung dieser Marerie. 
Texten besitzt. ${ }^{17}$ Jedenfalls hat Reginos Werk zunächst nur eine regionale Wirkung im 10. Jahrhundert entfalten können.

\section{Die Reformzeit des 11. Jahrhunderts}

Auf den Konzilien des 11. Jahrhunderts hat man zunächst die Priesterkinder von den Weihen ausgeschlossen. Eine solche Vorschrift scheint schon auf dem Konzil von Pavia 1014 ein Verhandlungsgegenstand gewesen zu sein, wurde dort aber nach einer in der Praefatio Papst Benedikts VIII. zu diesem Konzil enthaltenen Mitteilung auf eine spätere Synode verschoben. ${ }^{18}$ Die erste eindeutige Konzilsbestimmung findet sich auf dem Reformkonzil von Bourges 1031, welches die Priestersöhne und alle aus einer nicht legitimen Ehe stammenden Sprößlinge von allen Weihen beginnend mit der Subdiakonatsweihe ausschließt. ${ }^{19}$ Das Konzil beruft sich zur Begründung zunächst auf das im Deuteronomium enthaltene Verbor für Bastarde, den Tempel des Herrn zu betreten, außerdem auf die Diskriminierung des Bastards im weltlichen Recht in bezug auf Erbschaft und Zeugnisfähigkeit. ${ }^{20}$ Aus der ausführlichen Begründung für den Konzilskanon läßt sich erkennen, daß das Verbot durchaus noch als neuartig empfunden wurde; es entsteht aufgrund einer Angleichung eines kirchlichen Bewertungstandards an den der säkularen Umwelt, wobei man aber durch den Rückgriff auf das Alte Testament die Veränderung der Wertsetzungen zugleich als religiös geboten darstellen konnte. Es wäre deshalb falsch, im defectus natalium so etwas wie eine Kapitulation der Kirche vor den Wertsetzungen der Umwelt zu sehen. In einer Epoche, in der Priestersöhne zahlreich waren und in ihrer sozialen Situation in erster Linie eine kirchliche Karriere anstreben mußten, hat sich das Verbot des Konzils von Bourges zunächst keineswegs allgemein durchsetzen können. In einem Brieffragment Papst Alexanders II., das über die Collectio Britannica überliefert und zu Gratian gelangt ist ${ }^{21}$, legt der Papst fest, daß beim Vorhandensein sonstiger Tugenden jemand auch als Priestersohn für eine kirchliche Karriere in Frage komme; die Zulassung scheint hier auf eine Ermessensentscheidung hinauszulaufen. ${ }^{22}$

17 Vgl. Peter Landau, Gefälschtes Recht in den Rechtssammlungen bis Gratian, in: Fälschungen im Mitrelalter, Teil 2: Gefälschte Rechtstexte. Der bestrafte Fälscher (MGH Schriften, Bd. 33/II, Hannover 1988) 11-49, hier 22-25.

18 Conc. Ticinense ao. 1014, Johannes Domenicus Mansi, Sacrorum conciliorum nova et amplissima collectio (künftig: Mansi) (Paris 1902, ND Graz 1960) Bd. 19, col. 346: Taceo nunc de filiis, qui ingenuo clerico et libera matre, licet contra leges, nascantur: contra quos alia manu erit agendum, et in proxima Synodo consilio altiore tractandum.

19 Conc. Bituricense ao. 1031, c. 8, Mansi, (wie Anm. 18), col. 504: Ut filii presbyterorum, sive diaconorum, sive subdiaconorum, in sacerdotio, vel diaconatu, vel subdiaconatu nati, nullo modo ulterius ad clericatum suscipiantur.

20 Ebd.: quia tales, et omnes alii qui de non legitimo coniugio sunt nati, semen maledictum in scripturis divinis appellantur (= Deut. 23.2) nec apud saeculares leges haereditari possunt, neque in testimonium suscipi.

2) 56.12 (= JL 4611).

22 56.12: Nam pro eo, quod filius sacerdotis dicitur, si ceterae virtutes in eum conveniant, non reicimus, sed suffragantibus meritis connivendo eum recipimus. Vgl. hierzu das Dictum Gratiani: Hoc autem, quod de fliis sacerdotum dicitur, ex dispensatione ecclesiae introductum videtur. 
Das Konzil von Poitiers 1078 legt dann ein Verfahren fest, mit dem man die Eignung eines Priestersohns oder eines sonstigen Nichtehelichen festellen könne: Die Zulassung zu den höheren Weihen setze bei dieser Personengruppe voraus, daß sie zunächst als Mönche oder Regularkanoniker eine Art Probezeit absolviert haben. ${ }^{23}$ Diese Norm, die von einem unter dem Vorsitz eines Legaten Gregors VII. abgehaltenen Konzil stammte, ist durch die spätere Aufnahme in den Liber Extra zur Grundlage für die ganze Materie geworden, wie es im vorigen Jahrhundert der Kanonist Phillips ausdrückte ${ }^{24}$; die Regelung des Konzils von Poitiers wurde 1095 von dem unter Papst Urbans II. Vorsitz abgehaltenen Konzil von Clermont erneut aufgegriffen und wiederholt ${ }^{25}$; desgleichen von dem Konzil von Melfi $1089 .{ }^{26}$ Die von Alexander II. entwickelte Ermessensregel wurde ebenfalls in den Kanonessammlungen der gregorianischen Reform tradiert, besonders in einem bei Gratian Papst Urban II. zugeschriebenen Brief nach Tours zu einer Bischofswahl in Le Mans - eine Dekretale, die aber vielleicht bereits von Alexander II. stammt. ${ }^{27}$

In einem ebenfalls über die Collectio Britannica überlieferten Brief Urbans II. an den Bischof von Lüttich beantwortet der Papst die Anfrage des Bischofs Patronus, der aus einem ehebrecherischen Verhältnis stammte und beim Papst anfragte, ob er unter diesen Umständen im Amt verbleiben könne. Der Papst sieht in einer solchen Herkunft zwar eine Sünde, glaubt aber vor allem auf der Basis von Zeugenaussagen und der Information durch den Bischof selbst zu wissen, daß diesem sonstige Verfehlungen nicht vorzuwerfen seien. Daraus zieht Urban II. die Konsequenz, daß er den Bischof vom Makel der nichtehelichen Geburt befreit und ihn ausdrücklich in seinem Amt bestätigt. ${ }^{28}$ Wir haben also zur Zeit Urbans II, im kanonischen Recht bereits drei Rechtsgrundsätze: 1) Priestersöhne und unehelich Geborene sind grundsätzlich von den höheren Weihen ausgeschlossen; 2) sie können durch Leben in Klöstern oder Kanonikerstiften den ihnen anhaftenden Makel selbst zum Verschwinden bringen; 3) der Papst kann in einer Art Bestätigungsverfahren den Unehelichen zu kirchlichen Würden zulassen.

Diese Rechtslage spiegelt sich nun auch in den zeitgenössischen Rechtssammlungen, von denen ich hier nur die verbreitetste, nämlich die Panormie des Ivo von Chartes, herausheben möchte. Ivo nimmt in die Panormie vier Bestimmungen zu dem hier erörterten Problem auf. Er bringt zunächst den Kanon des Konzils von Melfi, der seinerseits die Bestimmung des Konzils von Poitiers wiederholt hat. ${ }^{29}$ Er fügt dann ein Exzerpt aus dem

23 Conc. Pictavense ao. 1078 c. 8, Mansi (wie Anm. 18), Bd. 20, col. 498: Ut filii presbyterorum et ceteri ex formicatione nati ad sacros gradus non provehantur, nisi aut monachi fiant, aut in congregatione canonica regulariter viventes.

24 Im Liber Extra X 1.17.1. Vgl. Georg Phillips, Kirchenrecht, Bd. 1 (Regensburg 1855, ND Graz 1959) 533.

25 Conc. Claromontanum ao. 1095 c. 9 - bei Mansi (wie Anm. 18), Bd. 20, col. 817, c. 11 - nach der Edition von Robert Somerville, The Councils of Urban II, vol. 1: Decreta Claromontensia (Amsterdam 1972) 76: Ut nulli filii concubinarum ad ordines vel ad aliquos honores ecclesiasticos promoveantur, nisi monachus vel canonice vixerit in ecclesia.

26 Conc. Melfitanum ao. 1089 c. 14, Mansi (wie Anm. 18), Bd. 20, col. 724 = Decr. Grat. 56.1.

$27 \mathrm{JL} 4610$ (=56.13). Hierzu vgl. Francis Gossman, Pope Urban II and Canon Law (CLS 403, Washington 1960) $118 f$. Im folgenden zitiert: Gossman, Urban II.

28 JL 5390 (= 56.14). Zu diesem Text vgl. auch Gossman, Urban II (wie Anm. 27), 28.

29 Ivo, Pan. 3.51 (PL 161, col. 1242) 
Liber Pontificalis hinzu, das er zuerst in seinem berühmten Prolog verwendet hat und das der gelehrte Kanonist Ivo offenbar selbst aus verschiedensten Papstbiographien zusammengestellt hat, um den Nachweis zu führen, daß in der Geschichte der Päpste häufig Söhne von Priestern oder gar Bischöfen zu Nachfolgern Petri aufgestiegen seien. ${ }^{30}$ Dieses interessante Exzerpt setzt die genaue Durcharbeitung des Liber Pontificalis voraus und kann nur als Versuch bewertet werden, Gegenargumente gegenüber den konziliaren Verboten zu sammeln. Es folgen sodann in Ivos Panormie die beiden Brieffragmente Alexanders II. und Urbans II. aus der Britannica, die sowohl für Priestersöhne als auch für sonstige Nichteheliche Ausnahmeerlaubnisse aufzeigten. ${ }^{31}$ Die Gesamttendenz bei Ivo ist eindeutig: Das rigorose Verbot der gregorianischen Reform wird aufgenommen, aber durch die Möglichkeit päpstlicher Dispense und außerdem den Aufweis einer dem Verbot nicht entsprechenden kirchlichen Praxis bei der Besetzung des päpstlichen Stuhls gewissermaßen stark relativiert, so daß sich die'sonst bei Ivo beobachrete vermittelnde Haltung zwischen radikalen Reformideen und diesen widersprechender Tradition auch auf unserem Gebiet feststellen läßt.

\section{Gratian}

Etwa fünfundvierzig Jahre nach Ivo wird das Problem der Weihe von Illegitimen von Gratian in seinem Dekret erneut ausführlich behandelt. Er widmet ihm die Distinctio 56. An den Anfang stellt er die prinzipielle Aussage: Presbiterorum etiam filii ad sacra offitia non sunt admittend $3^{32}$ - wobei er sich primär, ähnlich wie Ivo, auf das Konzil von Melfi stützt. Im Verlauf der Diskussion problematisiert Gratian dann aber zunächst den Ausschluß der Priestersöhne von den Ämtern, da er die enge Auslegung erwägt, das Verbot würde überhaupt nur für diejenigen Sprößlinge gelten, die die sexuelle Liberrinage ihrer Väter nachahmen würden. Handelte es sich um sittlich einwandfreie Nachkommen, so könnten sie nicht nur zu Bischöfen, sondern sogar zu Päpsten aufsteigen. ${ }^{33}$

Gratian verbindet dann dieses Liber-Pontificalis-Exzerpt Ivos mit einer ganzen Reihe von Kirchenväterstellen, nach denen die Vergehen der Eltern den Nachkommen nicht anzurechnen seien und vielmehr die uneheliche Geburt kein Hinderungsgrund für das Priesteramt sein dürfe. Insgesamt sind es sieben patristische Texte, die in dieser Geschlossenheit niemals vorher in der kanonistischen Tradition vereinigt waren. ${ }^{34}$ Das massivste Argument für die Zulassung der Unehelichen zum Priesteramt ist dabei die von Hieronymus in diese Diskussion eingeführte Abstammung Jesu Christi selbst, der nach Matthäus 1.3 von der unehelichen Verbindung des Juda mit der Thamar nach Genesis

30 Ebd. 3.52 (= d.p. 56.2).

31 Ebd. 3.53 (= JL 4610) und Pan. 3.54 (= JL 5390).

32 d.a. 56 pr.

33 d.p. 56.1: Sed hoc intelligendum est de illis, qui paternae incontinentiae imitatores fuerint. Verum si morum bonestas eos commendabiles fecerit, exemplis et auctoritate non solum sacerdotes, sed etiam summi sacerdotes fieri possunt.

34 56.3-9. 
38.25 abstammte. ${ }^{35}$ Gegen diese biblischen und patristischen Autoritäten führt Gratian dann aber zunächst den heiligen Bonifatius ins Feld, der in einem Brief an einen englischen König ausgeführt hatte, daß sexuelle Vergehen ganze Völker verderben könnten, also die Laster der Eltern sehr wohl auf die Nachkommen übergingen ${ }^{36}$; dieselbe These wird dann noch durch einen Hinweis auf eine Bibelstelle im Liber Sapientiae Salomonis begründet. ${ }^{37}$ Die historischen Fälle von Päpsten mit Klerikern als Vätern werden von Gratian mit der Argumentation entschärft, daß sie vor der Einführung des Zölibatsgebots für Priester und Diakone liegen würden und somit nicht für die Zulassung von Priestersöhnen zum geistlichen Dienst beweiskräftig seien. ${ }^{38} \mathrm{Da}$ das Zulassungsverbot auf biblischen Sätzen gegen den Ehebruch beruht, müssen auch die Söhne von Ehebrechern ebenso wie die Priestersöhne behandelt werden - das Zulassungsverbot kann sich also nicht nur gegen die Priesterkinder richten. ${ }^{39}$ Die Ausnahmeerlaubnisse bei Alexander II. und Urban II. werden von Gratian aufgenommen und so gedeutet, daß es sich hier um Einzelfallentscheidungen aufgrund der Dispensationsgewalt der Päpste handle; man dürfe deshalb diese Sätze nicht als zu verallgemeinernde Rechtsregeln verstehen. ${ }^{40}$

Im ganzen ist bei Gratian bereits ein Rechtszustand dargestellt, der im wesentlichen die Strukturelemente des klassischen kanonischen Rechts enthält. Das Weiheverbot wird als allgemeine Norm in den Mitrelpunkt gestellt; abweichende Quellenaussagen werden zum Teil mit historischen Argumenten neutralisiert. Zulassungen trotz der Verbote werden systematisch als Ausnahmen im Verhältnis zu Regeln gedeutet. Insgesamt ist der juristische Fortschritt im Vergleich zu Ivo von Chartres unverkennbar; aber auch das stärkere Ernstnehmen eines Verbots, das bei dem Bischof von Chartres noch erheblich relativiert worden war. Die biblische Begründung des Verbots hat bei Gratian einen hohen Stellenwert.

35 56.8. Quelle Gratians könnte hier Anselm von Lucca, Collectio Canonum 6.136. Hrsg. v. Friedrich Thaner (Innsbruck 1906-1915, ND Aalen 1965) 333 gewesen sein.

36 56.10. Es handelt sich um ein Stück aus Brief 73 des Bonifatius an König Aethelbald von Mercien; vgl. Bonifatii Epistulae. Hrsg. v. Reinhold Rau (Darmstadt 1968) (Freiherr vom Stein-Gedächtnisausgabe, Quellen zur Gesch. des Mittelalters 4b) 220-222. Gratian dürfte hier als Quelle die Collectio Tripartita Ivos von Chartres benurzt haben (Trip. I. 58.1).

37 d.p. 56.13: Adulterorum filli abominatio sunt Domino. Der Satz faßt sinngemäß Liber Sapientiae 3.16-19 zusammen.

38 d.p. 56.13: Cum ergo ex sacerdotibus nati in summos Pontifices supra leguntur esse promoti, non sunt intelligendi de fornicatione, sed de legitimis coniugiis nati, que sacerdotibus ante probibitionem ubique licita erant, et in orientali ecclesia usque hodie eis licere probatur.

39 d.p. 56.13, \$1: Sed sicut supra dictum est de presbiterorum filiis, ita et hoc de filiis adulterorum intelligendum uidetur, ut illi dicantur esse abhominatio Domino, ad quos paterna flagitia hereditaria successione descendunt.

40 d.p. 56.12: Hoc autem, quod de filiis sacerdotum dicitur, ex dispensatione ecclesiae introductum uidetur, et quod ex dispensatione introducitur, ad consequentiam regulae trahi non poterit. 


\section{Die Dekretalen und die Kanonistik}

Kommen wir von Gratian zum Dekretalenrecht des 12. Jahrhunderts, das uns vor allem in den Dekretalen Alexanders III. und seiner unmittelbaren Nachfolger überliefert ist, so ergibt sich gegenüber dem sehr klaren Bild bei Gratian zunächst die überraschende Feststellung, daß sich die Dekretalen fast ausschließlich mit dem Problem der Versorgung von Priestersöhnen mit kirchlichen Ämtern und Pfründen befassen, also einer Problematik des kirchlichen Amtsrechts und der Korruption, während der theologisch begründete personale Aspekt demgegenüber fast völlig zurücktritt.

Das Dekretalenrecht ist in diesem Bereich vor allem durch die Collectio Appendix Concilii Lateranensis beeinflußt worden, die gegen Ende des Pontifikats Lucius' III. in England zusammengestellt wurde und einen Titelabschnitt mit der Rubrik: De ordinatione filiorum sacerdotum et eisdem in ecclesiis paternis non tolerandis enthielt. ${ }^{41}$ Von den zehn Dekretalen dieses Titels sind acht über die Bambergensis und die Compilatio I in den Liber Extra gelangt und machen somit fast die Hälfte des Titels De filiis presbyterorum ordinandis vel non des Liber Extra aus (X 1.17.2-9). ${ }^{42}$ Es handelt sich hier in jedem Fall um Rechtsstreitigkeiten, ob ein nach der Priesterweihe - in sacerdotio - erzeugter Priestersohn seinem Vater in dessen Benefizium nachfolgen dürfe. Papst Alexander III., von dem sämtliche Dekretalen stammen, bekräftigt zunächst ein generelles Sukzessionsverbot für Priestersöhne, das auch zur Folge haben soll, daß eine Einsetzung aufgrund eines Betrugs, weil der Papst nämlich über die Verwandtschaft getäuscht wurde, Dispens von der Illegitimität erteilte und dann unwissend an den Illegitimen die väterliche Kirche vergab, rückgängig zu machen sei. ${ }^{43}$ Jedoch betont der Papst ausdrücklich, daß eine wissentlich erfolgte Einsetzung eines Priestersohns durch den Bischof in die väterliche Kirche keineswegs später aufgehoben werden dürfe - eine solche Einsetzung ist zwar unerlaubt, bleibt aber wirksam. ${ }^{44}$ Das Verbot wird von Alexander III. aber schon insofern relativ eng umgrenzt, als nur die unmittelbare Nachfolge untersagt ist - sofern die Pfründen zwischenzeitlich einem Dritten gehörte: media intercessit persona-, ist die Nachfolge durchaus erlaubr. ${ }^{45}$ Bei unmittelbarer Sukzession kann der Priestersohn in einem summarischen Verfahren ohne Zeugenvernehmung entfernt werden, wenn die Abstammung manifest ist. ${ }^{46}$

Das Verbot der Erbfolge in Kirchen kann wie viele andere Verbote des positiven kanonischen Rechts durch Dispens außer Kraft gesetzt werden; eine generelle Vollmacht zur

41 Appendix Concilii Lateranensis Tit. 19; vgl. Emil Friedberg. Die Canones-Sammlungen zwischen Gratian und Bernhard von Pavia (Leipzig 1897, ND Graz 1958) 64. Im folgenden zitiert: Friedberg, Canones-Sammlungen.

42 App. $19.3=$ Bamb. $23.3=1$ Comp. 1.9.2 = X 1.17.2; App. $19.4=$ Bamb. $23.4=1$ Comp. $1.9 .3=\mathrm{X}$ 1.17.3; App. 19.1. = Bamb. 23.1 = 1 Comp. 1.9.4 = X 1.17.4; App. $19.5=$ Bamb. $23.5=1$ Comp. $1.9 .6=$ X 1.17.5; App. 19.6 = Bamb. 23.6 = 1 Comp. 1.9.7 = X 1.17.6; App. $19.8=$ Bamb. $23.7=1$ Comp. 1.9.8 = X 1.17.7; App. 19.9 = Bamb. $23.8=1$ Comp. $1.9 .10=$ X 1.17.8; App. $19.10=$ Bamb. $23.9=1$ Comp. 1.9.11 = X 1.17.9. Vgl. zu diesen Parallelstellen Friedberg, Canones-Sammlungen (wie Anm. 41), 175.

$43 \times 1.17 .2$ (JL 13982).

$44 \times 1.17 .5$ (JL 13995).

$45 \times 1.17 .7$ (JL 13248).

$46 \times 1.17 .10$ (JL 13815). 
Erteilung solcher Dispense vergibt Alexander in einer Dekretale an den Erzbischof von York. ${ }^{47}$ Eine weitere Einschränkung besteht darin, daß die Sohnesnachfolge nur dann ausgeschlossen ist, wenn der Vater die Pfründe auf Dauer innegehabt hat; sie betrifft nicht den Fall, daß der Vater in der umstrittenen Kirche überhaupt einmal geistliche Funktionen ausübte. ${ }^{48}$ In allen Fällen ist vorausgesetzt, daß der die väterlichen Pfründen beanspruchende Kleriker bereits ordiniert war; von einer Versagung der Ordination oder auch von einer Dispenserteilung vor der Ordination ist in diesen Dekretalen nirgends die Rede. Nur eine einzige dieser Dekretalen behandelt einen Fall, bei dem es nicht um "Familienkirchen“ geht - ein Priestersohn wird von einem Patron für die Patronatskirche präsentiert; Bischof Bartholomäus von Exeter weigert sich, ihn in die Pfründen einzusetzen. Der Kandidat hat aber bereits die Subdiakonatsweihe empfangen, so daß er eine Art Versorgungsanspruch hat. Der Papst ordnet an, daß zwar ein anderer die Pfründen formell erhalten solle, aber der vom Patron vorgesehene Subdiakon mit der Hälfte der Einkünfte der betreffenden Kirche abzufinden sei. ${ }^{49}$ Hier werden vom Papst die Versorgungsinteressen der Illegitimen im Klerus sehr ernst genommen; aber auch diese Dekretale enthält nicht etwa ein Verbot, Illegitime zu weihen, obwohl beim Aufrichten einer solchen Schranke das ganze Versorgungsproblem gar nicht erst entstanden wäre.

Die Akzentsetzung liegt in dieser Zeit eindeutig auf der Verhinderung der Erblichkeit, nicht etwa in der Unterbindung der Ordination. Das zeigt sich besonders deutlich an einer Dekretale Alexanders III., die in der Appendix-Sammlung zuerst in einen späteren Anhang aufgenommen ist. ${ }^{50}$ Hier schreibt Alexander III. dem Erzbischof von Canterbury, daß es eine Nachfolge in Kirchen ihrer Väter auch für die legitimen Priestersöhne nicht geben dürfe, also für diejenigen, die vor der Priesterweihe des Vaters geboren seien - sive geniti sint in sacerdotio sive non. Soweit ersichtlich, hat Alexander III. nur in einer einzigen nach Tours gerichteten Dekretale auf eine Anfrage des dortigen Erzbischofs hin erklärt, daß der Erzbischof Nichteheliche und Unfreie nicht zu den Weihen zulassen solle. ${ }^{51}$ Diese Dekretale ist von den Sammlern jedoch lange Zeit nur zur Begründung des Ordinationsverbots für Unfreie herangezogen worden, so in der Collectio Parisiensis II und in der Collectio Bambergensis ${ }^{52}$ - in ihrer prinzipiellen Bedeutung für Illegitime scheint sie zuerst Bernhard von Pavia erfaßt zu haben. ${ }^{53}$

Im Dekretalenrecht ist das Prinzip der Irregularität aufgrund des defectus natalium erst verhältnismäßig spät proklamiert worden, obwohl im Dekret Gratians eine eindeutige prinzipielle Stellungnahme nur indirekt erschlossen werden konnte, und insofern ein

$47 \times 1.17 .9(\mathrm{JL} 13881)$.

$48 \times 1.17 .8(\mathrm{JL} \mathrm{14145)}$.

49 $\mathrm{X} 1.17 .6(\mathrm{JL} 14217)$.

so App. $50.60=2$ Comp. 1.9.1. $=$ X 1.17.11 (JL 13802). Hierzu Friedberg, Canones-Sammlungen (wie Anm. 41), 83.

51 X 1.18.5 (JL 14121).

52 Par. $17.4=$ App. $26.19=$ Bamb. $19.4=1$ Comp. 1.10.5 = X 1.18.5. Zum Vorkommen dieser Dekretale in den genannten Dekretalensammlungen vgl. Friedberg, Canones-Sammlungen (wie Anm. 41), 35 und 99.

53 Bernardus Papiensis, Summa Decretalium, Lib. 1, Tit. 9. Hrsg. v. Ernst Adolph Theodor Laspeyres (Regensburg 1860, ND Graz 1956) 11: De spuriis non ordinandis habes infra tit. prox. Consuluit; im folgenden zitiert: Bernardus Papiensis, Summa Decretalium. 
Bedürfnis nach einer eindeutigen gesetzlichen Regelung aus der Betrachtungsweise eines modernen Juristen an sich hätte bejaht werden müssen. Rufinus verstand die dialektisch bei Gratian nicht eindeutig aufgelösten Widersprüche in dem Sinne, daß Gratian die Zurechnung der Fehler der Eltern bei den Söhnen gerade abgelehnt habe, sofern die Priestersöhne sich als religiosi bewährt hätten - hier wird Gratian mit der Tendenz der vom Magister rezipierten patristischen Texte identifiziert und schließlich gesagt, daß es sich im Grunde bei der Abstammung nur um ein rechtlich nicht bedeutsames, wohl moralisch zu verstehendes Hindernis handle: Hoc denique impedimentum non impediebat, sed impedire videbatur. ${ }^{54}$

Die Rezeption einer Grundsatznorm über den Ausschluß von Priestersöhnen und sonstiger Nichrehelicher von den heiligen Weihen scheint erst in den achtziger Jahren in den Dekretalensammlungen erfolgt zu sein, wobei sich ohne Spezialuntersuchung kaum klären läßt, ob die Kanonistik hier den Dekretalensammlern schon vorher eine Anleitung gab. Die Grundsatznorm war der bereits erwähnte Kanon 8 des Konzils von Poitiers 1078 mit der klaren Aussage: ut filii presbyterorum et ceteri ex fornicatione nati ad sacros ordines non promoveantur. Dieser Konzilskanon mit der generellen Erwähnung der Nichtehelichen, nicht nur der Priestersöhne, war von Gratian nicht berücksichtigt worden, und hatte auch schon bei Ivo von Chartres keine Rolle gespielt; er wird erst von den Dekretalensammlern entdeckt und bildet seitdem eigentlich die "Grundlage für die ganze Materie" (Phillips). Das Konzil von Poitiers taucht nun in den Dekretalensammlungen zuerst um 1185 in der Collectio Lipsiensis aufs; von dort gelangt es in die Compilatio I, wo die einzelnen Konzilscanones zuerst auf die für sie in Frage kommenden Titel verteilt sind; also auch der Kanon über die Illegitimen. Es ist demnach Bernhard von Pavia, der für die Rezeption dieser Grundsatznorm im kanonischen Recht verantwortlich ist - denn Bernhard stellte sowohl die Lipsiensis als auch die Compilatio I zusammen. ${ }^{56}$ Indem der Poitiers-Kanon mit den Dekretalen Alexanders III. in einem Titel zusammengebracht und dabei sogar an die Spitze gerückt wurde, erhielten die Stellungnahmen Alexanders III. zu den Problemen der Vererbung von Benefizien, zu den Familienkirchen, plötzlich eine ganz neue prinzipielle Bedeutung. Bernhard kommentierte den von ihm zusammengestellten Titel der Compilatio I in seiner Summa decretalium und betonte, daß es sich hier generell um ein vitium originis für die Priesterweihe handle. Bernhard macht auch eine prinzipielle Aussage über den Zweck des Ordinationsverbots: causa probibitionis est dignitas sacerdotalis. ${ }^{57}$ Damit wird vom Sakrament der Priesterweihe her eine Begründung versucht, also anders als in den früheren Dekretalen ein letztlich theologischer Gesichtspunkt in die Diskussion eingeführt.

54 Rufinus, Summa decretorum ad 56.14. Hrsg. v. Heinrich Singer (Paderborn 1902, ND Aalen 1963) 150: Impedimentum quoddam, dico, non vitium; alioquin adversaretur ei, quod supra dixit Ieronimus, cap. Nasci. Hoc denique impedimentum non impediebat, sed impedire videbatur.

55 Collectio Lipsiensis 52.2 - vgl. Emil Friedberg, Quinque Compilationes antiquae nec non Collectio Canonum Lipsiensis (Leipzig 1882, ND Graz 1956) 203. In der Lipsiensis erscheinen die Texte des Konzils von Poitiers noch in einem Titel De iure patronatus in ecclesiis clericis concessis a laicis.

$56 \mathrm{Vgl}$. Peter Landau, Die Entstehung der systematischen Dekretalensammlungen und die europäische Kanonistik des 12. Jahrhunderts, in: ZRG KA 65 (1979) 120-148, hier 135f.

57 Bernardus Papiensis, Summa Decretalium (wie Anm. 53), 11. 
Die stärkere Betonung des Ausschlusses der Illegitimen von den höheren Weihen in Bernhards Breviarium, der Compilatio I, führt dann bereits während des Pontifikats Clemens' III. dazu, daß ein betroffener Priestersohn, der nach der Weihe des Vaters in einer vor der Weihe geschlossenen legitimen Ehe erzeugt wurde, eine Dispens für die Ordination erbittet. Die entsprechende Dekretale ist über die Sammlung des Kanonisten Gilbert um 1202 in das Dekretalenrecht gelangt ${ }^{58}$ und ist in diesem Bereich das zeirlich erste Quellenzeugnis für Einholung und Gewährung einer Dispens vor der Weihe wegen des defectus natalium. Daraus ergibt sich, daß der Defekt als Weihehindernis erst gegen Ende des 12. Jahrhunderts in der Rechtswirklichkeit der Kirche ansatzweise akzeptiert war.

$\mathrm{Daß}$ am Anfang des 13. Jahrhunderts schon das Gerücht einer nichtehelichen Geburt bzw. der Vaterschaft eines Priesters dazu führen konnte, daß sich die Bischöfe weigerten, an davon Betroffenen die Priesterweihe zu vollziehen, läßt sich aus einer Dekretale Innozenz' III. an den Bischof von Florenz vom 6.2.1207 entnehmen. ${ }^{59}$ Einem Diakon der Florentiner Kirche war die Weihe verweigert worden, weil ihm von mißgünstigen Nebenbuhlern uneheliche Geburt vorgeworfen wurde. Die Frage ließ sich nicht durch Beweisaufnahme klären, so daß der Papst empfiehlt, dem übel Beleumundeten einen Reinigungseid abzunehmen, obwohl es sich hier gar nicht um einen Deliktsvorwurf handelte. Der Reinigungseid wird also hier als Prozeßmittel eingesetzt, um ein Dispensverfahren vermeiden zu können, das für den Diakon nach Ablegung dieses Eides nicht mehr nötig ist. Abgesehen davon, daß auch hier im Dekretalenrecht eine präventive Verwendung des Hindernisses vor der Ordination belegt ist, geht die Tendenz insgesamt dahin, die Zahl von Dispensverfahren offenbar nicht übermäßig auszudehnen, damit die Kirche mit dem durch ihre Rechtsordnung geschaffenen Problem fertig werden konnte.

Das vierte Laterankonzil hat im Kanon 31 den Verbotskatalog in bezug auf Pfründenempfang für Priestersöhne dann noch insofern gegenüber den Dekretalen Alexanders III. erweitert, als solche Personen nicht neben ihren Vätern Kanoniker in demselben Kapitel werden konnten. Verbotswidrige Einsetzung auf Kapitelspfründen sollte nichtig sein. ${ }^{60}$ Diese Bestimmung ergänzt die Verbote Alexanders III. und verfolgt offenbar den gleichen Zweck, eine familiäre Ausnutzung von Kirchen zu verhindern.

In der späteren Dekretalengesetzgebung sind vor allem zwei Dekretalen Gregors IX.61 und Bonifaz' VIII. ${ }^{62}$ bedeutsam. Gregor IX. reserviert in einem Schreiben an den Erzbischof von Tours, das 1234 in den Liber Extra aufgenommen wurde, für den Papst die Dispensvollmacht, sofern es sich um die Besetzung ranghoher Pfründen wie Dignitäten und Personate sowie von allen Seelsorgebenefizien handelt. Hier ist demnach indirekt

s8 Collectio Gilberti 1.10.3 = 2 Comp. 1.9.5 = X 1.17.14 (JL 16633). Zu Gilbert vgl. Rudolf v. Heckeh Die Dekretalensammlungen des Gilbertus und Alanus nach den Weingartener Handschriften, in: ZRG KA 29 (1940) 116-357, hier 186.

$59 \times 5.34 .14$ (Po. 3001).

604 Conc. Lateranense c. 31. Hrsg. v. Antonio Garcla y Garcla, Constitutiones Concilii quarti Lateranensis una cum Commentariis glossatorum, Città del Vaticano 1981 (= Monumenta Iuris Canonici, Ser. A, Bd. 2) $75=\mathrm{X}$ 1.17.16.

$61 \mathrm{X} 1.17 .18$ (Po. 9551).

62 VI 1.11.1. Hrsg. v. Friedberg col. 977. 
ausgeschlossen, daß der Papst auch bei der normalen Priesterweihe eines Illegitimen Dispens erteilen müsse; insofern besteht offenbar eine primäre Zuständigkeit des Diözesanbischofs. Ganz anders werden jedoch 60 Jahre später die Zuständigkeiten im Liber Sextus Bonifaz' VIII. verteilt: Danach ist der Papst zuständig für alle Dispense bei der Erteilung höherer Weihen, während die Kompetenz des Bischofs darauf reduziert ist, daß er die Illegitimen, die bei Bonifaz zum ersten Mal in der Gesetzgebung mit dem technischen Begriff des defectus natalium erfaßt werden, nach einer Dispens zu den niederen Weihen zulassen darf. Das Weihehindernis umfaßt jetzt auch die niederen Weihen, die in den Verbotsnormen vor 1234 nirgends erwähnt werden. Die Frage, ob der Bischof überhaupt Illegitime für die höheren Weihen dispensieren dürfe, erscheint noch Bernhard von Botone in der Glossa ordinaria zum Liber Extra als ungeklärt, die von ihm selbst gestellte Frage: Sed numquid episcopi possunt dispensare cum talibus illegitime natis in simplici beneficio, et ut promoveantur ad sacros ordine sine aliqua praelatione ${ }^{x 3}$ beantwortet er nicht eindeutig.

Die Rechtslage bezüglich der Dispensvollmachten war offenbar während des ganzen 13. Jahrhunderts in der Kanonistik umstritten. Goffredus behandelt in seiner Dekretalensumme das Problem sehr ausführlich und stellt bereits fest, daß sich ein ausdrückliches Dispensverbot für Bischöfe im positiven Recht nicht finden lasse: Non enim invenitur probibitum episcopis circa ordines et simplicia beneficia cum talibus dispensare unde intelligitur eis permissum. ${ }^{64}$ Nach der in der Summa aurea des Hostiensis wiedergegebenen Diskussion hat offenbar vor allem Damasus die These vertreten, daß eine Dispens zur Erlangung höherer Weihen allein der Papst erteilen könne; Hostiensis bezweifelt diese Ansicht, da er ein gesetzliches Verbot nicht finden könne, folgt also der Argumentation des Goffredus. ${ }^{65}$ Schon vor Goffredus hat Raymund von Peñafort in seinem Jugendwerk, der Summa Iuris, um 1220 einmal das Verbot der Weihe von Illegitimen ohne weitere Begründung auch auf die niederen Weihen bezogen, indem er ausführte, daß der Bischof für die niederen und auch für die höheren Weihen Dispens erteilen dürfe, der Papst aber allein für die Dispens bei der Bischofsweihe zuständig sei. ${ }^{66}$ Die Ausdehnung des Verbots auf die niederen Weihen steht offenbar in einem engen Zusammenhang mit einem erneuten Anknüpfen an den Text des Deuteronomium (Deut. 23.2), da Raymund ausdrücklich bemerkt: causa huius probibitionis est lex divina. Sowohl die Ausdehnung des Verbots auf die niederen Weihen als auch die enge Begrenzung der Dispensvollmacht der Bischöfe bei Bonifaz VIII. steht somit in der Tradition der kanonistischen Diskussion des 13. Jahrhunderts; der Liber Sextus bringt auf einem für die Praxis höchst wichtigen und in der Gesetzgebung nach Innozenz III. vernachlässigten Gebiet eine offenbar sehr erwünschte Rechtssicherheit. Daß die Regelung bei Bonifaz VIII. betont zentralistisch

63 Glossa ordinaria ad X 1.17 .18 v. sedis apostolice (Venetiis 1595) 210.

64 Goffredus Tranensis, Summa super titulis decretalium (Lyon 1519, ND Aalen 1968) ad X 1.17, f. 35rb.

65 Henricus de Segusio (Cardinalis Hostiensis), Summa (Lyon 1537, ND Aalen 1962) ad X 1.17, f. 37va: Quis possit dispensare? Dicunt quidam quod in magna et maiori episcopi... Damasus contra quia si hoc verum esset non concederetur eis speciali privilegio... Ergo dicas quod solus papa dispensat in maiori et maxima cum filio sacerdotis in seculo manente, sed in maiori potest dispensare episcopus, quia non invenio probibitum, immo potius concessum.

66 San Raymundo de Penyafort, Summa Iuris. Hrsg. v. José Rius Serra (Barcelona 1945), tit. XXII (94). 
ausfiel, ist in Anbetracht der Grundeinstellung dieses Papstes nicht verwunderlich; man muß jedoch betonen, daß der Papst hier wie wohl auch auf anderen Gebieten kein radikaler Neuerer war; der Liber Sextus steht durchaus in der Kontinuität einer in der Kanonistik angelegten Entwicklung. ${ }^{67}$

Die Geschichte des kanonischen Rechts auf dem Gebiet des Weihehindernisses der Illegitimen liefert ein gutes Beispiel für die insgesamt etwa gleichgewichtige Rollenverteilung von Wissenschaft und Gesetzgebung in der Zeit des klassischen kanonischen Rechts, wobei die prinzipiellen Anstöße häufig eher von der Kanonistik kommen, während es die Leistung des päpstlichen Dekretalenrechrs ist, so etwas wie eine Vermittlung von Prinzipien und Rechtswirklichkeit mit einem Instrument zu erreichen, für das man vielleicht den in der neueren juristischen Methodenlehre entwickelten Begriff der Fallnormen als adäquate Beschreibung verwenden kann. ${ }^{68}$

67 Zu diesem Thema vorläufig Tilmann Schmidt, Papst Bonifaz VIII. als Gesetzgeber, in: Proceedings of the Eighth International Congress of Medieval Canon Law (Città del Vaticano 1992) = Monumenta Iuris Canonici, Ser. C, vol. 9, 227-245. Von Schmidt sind zu diesem Bereich weitere Forschungen zu erwarten.

68 Wolfgang Fikentscher, Methoden des Rechts in vergleichender Darstellung, Bd. 4 (Tübingen 1977) 202268. 
